\title{
Warum und wie Industrielle Beziehungen den Parteieneffekt auf wohlfahrtsstaatliche Retrenchmentpolitik beeinflussen. Ein Vergleich zwischen den Niederlanden, Dänemark, Deutschland und Frankreich
}

\author{
Christine Trampusch
}

\begin{abstract}
Zusammenfassung: In der Analyse von Umverteilung sollten nicht nur staatliche, auf dem Territorialprinzip gründende Modelle Berücksichtigung finden, sondern auch funktionale, private, kollektive Einrichtungen, die von Tarifverträgen bereitgestellt werden. Am Beispiel der Altersvorsorge und auf der Grundlage eines die Differenz- mit der Konkordanzmethode kombinierenden und prozessanalytischen Vergleiches zeigt der Beitrag, dass in den Niederlanden und Dänemark der hohe Entwicklungsstand funktionaler Umverteilung den Parteieneffekt auf Retrenchmentprozesse erklärt, während umgekehrt der geringere Handlungsspielraum von politischen Parteien in Frankreich und Deutschland mit der traditionellen Dominanz territorial organisierter Umverteilung in eine Verbindung gebracht werden kann. Industrielle Beziehungen und Modelle funktionaler Umverteilung beeinflussen demnach den Einfluss von Parteien auf wohlfahrtsstaatliche Retrenchmentpolitik.
\end{abstract}

Schlüsselwörter: Parteien · Wohlfahrtsstaat · Industrielle Beziehungen · Prozessanalyse $\cdot$ Mill'sche Methoden

\begin{abstract}
In the analysis of redistribution, not only models based on the territority principle should be considered, but also functionally structured redistribution like collectively negotiated welfare benefits. Combining the methods of difference and agreement with process tracing the article shows that, in the Netherlands and Denmark, the high level of development of functionally arranged redistribution explains the strong party effect on processes of retrenchment in pension; on the other side, the smaller room for maneuver for political parties in Germany and France can be linked to the dominance
\end{abstract}

Prof. Dr. Christine Trampusch $(\bowtie)$

Assistenzprofessorin für vergleichende Politik, Institut für Politikwissenschaft (IPW),

Universität Bern, Lerchenweg 36, CH-3000 Bern 9

Tel.: +41 (0)316313750

Fax: $+41(0) 316318590$

E-Mail: christine.trampusch@ipw.unibe.ch 
of territorially organized redistribution. Industrial relations and models of functional redistribution affect the influence of political parties on welfare state retrenchment.

Keywords: Political Parties · Welfare State - Industrial Relations · Process Tracing · Mill's Methods

\section{Einleitung}

Umverteilung - der Transfer von Geld und Leistungen von besser gestellten auf schlechter gestellte Marktteilnehmer - setzt die Existenz nicht-marktlicher Institutionen voraus, mittels derer Ressourcen verteilt und Risiken unter den Mitgliedern einer Gruppe (oder zwischen Gruppen) gepoolt werden (Polanyi 1944: 43-55). Umverteilung ist solidarisches Verhalten. Sie beruht auf der Bereitschaft von Gruppenmitgliedern, für das Wohlergehen der Mitglieder einer Gruppe Opfer zu bringen. Wieviel in einem Wohlfahrtsstaat umverteilt wird, hängt nicht nur vom Markteinkommen, der Steuerpolitik oder von Merkmalen der Inanspruchnahme sozialer Leistungen ab. Das Ausmaß an Umverteilung wird vielmehr auch maßgeblich von der sozialen Konstitution der Gruppen und Umverteilungskoalitionen bestimmt.

In Anlehnung an Thomas Marshalls Differenzierung zwischen politischen, sozialen und industriellen Bürgerrechten lassen sich hinsichtlich der Gruppenkonstitution idealtypisch zwei Modelle unterscheiden: (1) Das „territoriale Modell“, in dem auf der Grundlage von politischen Bürgerrechten durch Einführung einer verpflichtenden Sozialversicherung oder von staatlichen Fürsorgeleistungen soziale Bürgerrechte geschaffen werden, und damit Ansprüche an den Staat, der die Umverteilung organisiert und die Ressourcen verwaltet und verteilt. (2) Das zweite Modell ist ein ,funktionales Modell“. Hier wird auf der Basis von industriellen Bürgerrechten umverteilt, indem Gewerkschaften sozialen Forschritt mit Hilfe von in Tarifverträgen vereinbarten sozialen Leistungen durchsetzen. Das funktionale Modell gewinnt vor allem in der Rentenpolitik an Bedeutung. So haben in einer Reihe von Ländern Gewerkschaften und Arbeitgeberverbände auf sektoraler Ebene Tarifverträge geschlossen, die Tarifrenten finanzieren und regulieren (Trampusch 2007).

In der politikwissenschaftlichen vergleichenden Wohlfahrtsstaatenforschung stellt die Frage des Ausmaßes wohlfahrtsstaatlicher Umverteilungspolitik und wie dieses Ausmaß erklärt werden kann, derzeit einen Fixpunkt der Literatur dar. Im Kontext der Diskussion darüber, ob ein wohlfahrtsstaatlicher Retrenchmentprozess stattfindet, und damit auch ein Abbau an Umverteilungspolitik und Solidarität, fokussieren viele Studien jedoch allein auf Umverteilung, die auf dem territorialen Modell beruht. Es werden Staatsausgaben, Leistungen staatlicher Sozialversicherungen und -programme in den Mittelpunkt gestellt. Damit geht einher, dass auf der Seite der unabhängigen Variable vor allem der Einfluss von Akteuren und Koalitionen der territorialen Interessenrepräsentation im politisch-administrativen Raum untersucht wird: Es wird gefragt, ob und welche (partei-)politischen Akteure staatliche Sozialleistungen kürzen. In diesem Zusammenhang haben fallorientierte Studien zu Reformprozessen in der Rentenpolitik darauf aufmerksam gemacht, dass der Parteieneffekt im internationalen Vergleich variiert. 
So haben Parteien in den Niederlanden und Dänemark im Vergleich zu Deutschland und Frankreich nicht nur einen größeren Einfluss auf rentenpolitische Reformen, ihnen ist in den letzten Jahren auch das Kürzen einfacher gefallen (Green-Pedersen 2001; Schludi 2005; Conceição-Heldt 2006: 192; Green-Pedersen und Lindbom 2006; Schulze und Jochem 2006; Palier 2007).

Mittels eines fallorientierten Vergleiches zwischen den Niederlanden, Dänemark, Deutschland und Frankreich zeigt dieser Artikel, dass der unterschiedliche Parteieneffekt in diesen Ländern mit einem unterschiedlichen Entwicklungsstand funktionaler Umverteilung, also tariflich organisierter Altersvorsorge, zusammenhängt. Er schlägt vor, in der Analyse des derzeitigen Wandels wohlfahrtstaatlicher Umverteilungspolitik sowohl auf der Seite der ,abhängigen Variable“ als auch auf Seite der ,unabhängigen Variable“ eine Ergänzung vorzunehmen: Nicht nur der Entwicklungsstand funktional strukturierter Umverteilung ist zu berücksichtigen, es ist auch zu untersuchen, welche Rückwirkungen tarifliche Altersvorsorge auf die Prozesspolitik der Reform staatlich organisierter Umverteilungspolitik hat.

Auf methodischer Ebene verknüpft der Artikel die kombinierte Anwendung von Konkordanz- und Differenzmethode (Mill 1874: 283-284; Skocpol und Somers 1980: 183) mit fallinternem process tracing. Er basiert auf einer historisch-institutionalistischen Argumentationslogik. Ich gehe in drei Schritten vor: Im ersten Schritt wird für die ausgewählten Fälle am Beispiel der staatlichen Rentenpolitik und von Tarifverträgen zur Altersvorsorge die territorial wie auch funktional strukturierte Umverteilung genauer beschrieben. In den folgenden beiden Schritten analysiere ich auf der Basis eines zweistufigen Ländervergleiches den Zusammenhang zwischen funktional strukturierter Umverteilung und Parteieneffekt. Der Vergleich zeigt die Bedeutung von Entwicklungspfaden auf und verbindet eine historische Analyse von Pfaden politischer und industrieller Opportunitätsstrukturen mit aktuellen politischen Reformpräferenzen. Die erste Stufe des Ländervergleiches ist historisch. Ausgehend von Thomas H. Marshalls Unterscheidung zwischen politischen und industriellen Bürgerrechten wird anhand der ausgewählten Fälle die These illustriert, dass die zeitliche Abfolge (Sequenz) der Institutionalisierung von politischen und industriellen Staatsbürgerrechten entscheidend dafür ist, bis zu welchem Grad Umverteilung auch auf dem funktionalen Prinzip beruht: In Ländern, in denen industrielle Bürgerrechte vor politischen Bürgerrechten institutionalisiert und genutzt wurden - wie Dänemark und die Niederlande - sind funktionale Modelle der Umverteilung entwickelter als in Ländern, wo die Sequenz genau anders herum verlief - wie Frankreich und Deutschland. Aufbauend auf der These der Bedeutung von Verläufen der Demokratisierung und Institutionalisierung der Arbeitsbeziehungen wird in der zweiten Stufe des Ländervergleiches eine zweite These entwickelt, deren Plausibilität wiederum durch die kombinierte Anwendung von Konkordanz- und Differenzmethode illustriert wird: In Ländern, in denen das funktionale Modell der Umverteilung Tradition ist und neben territorial organisierter Umverteilung einen festen Platz hat (Dänemark, Niederlande), bestimmen Parteien und der Parteienwettbewerb den Verlauf des Retrenchments staatlicher Sozialpolitik mehr als in Ländern, wo das funktionale Prinzip eine untergeordnete Rolle spielt (Frankreich, Deutschland).

Das Papier ist wie folgt aufgebaut: Der folgende zweite Abschnitt verortet meinen Artikel in der politikwissenschaftlich vergleichenden Wohlfahrtsstaatenforschung. Der 
Schwerpunkt wird dabei auf die Literatur zu Parteieneffekten und auf Studien zu rentenpolitischen Reformprozessen in den vier Ländern gelegt. Der dritte beschreibt für die Niederlande, Deutschland, Frankreich und Dänemark zum einen die Rentensysteme und zum anderen die Tarifverträge zur Altersvorsorge. Der vierte Abschnitt erläutert die Vergleichsstrategie. Die folgenden beiden Abschnitte führen den zweistufigen Ländervergleich durch. Der fünfte Abschnitt verdeutlicht, warum Verläufe der Demokratisierung und Institutionalisierung der Arbeitsbeziehungen für die Entstehung und Entwicklung funktionaler Umverteilung von Bedeutung sind und überprüft dies anhand der vier Länder. Der sechste Abschnitt untersucht die prozesspolitische Rückwirkung funktionaler Umverteilungseinrichtungen. Der siebte Abschnitt fasst zusammen und fragt, wie eine historisch-institutionelle Analyse von Pfaden politischer und industrieller Opportunitätsstrukturen mit neueren Konzepten der Analyse inkrementellen Institutionenwandels verknüpft werden kann.

\section{Retrenchment und Umverteilung: Ein Blick in die vergleichende Wohlfahrtsstaatenforschung}

In der derzeitigen politikwissenschaftlichen vergleichenden Wohlfahrtsstaatenforschung stehen zwei Diskussionen im Zentrum: erstens die Diskussion darüber, ob und in welchem Ausmaß ein Retrenchment - und damit auch ein Abbau von Umverteilungspolitik und Solidarität - stattgefunden hat; zweitens die Frage, welche politischen Faktoren Retrenchment-Prozesse bzw. deren Ausbleiben erklären (Starke 2006).

Lange Zeit stand der These eines Abbaus von Umverteilungspolitik und Solidarität (van Oorschot 1998) die Anti-These entgegen, dass Retrenchment nachwievor schwierig ist und Wohlfahrtsstaaten keineswegs auf dem Weg zu einem residualen Modell der sozialen Sicherung sind (Pierson 1994). Mittlerweile weisen jedoch sowohl fallorientierte, qualitative Studien wie auch quantitative Aggregatdatendanalysen darauf hin, dass staatliche Sozialpolitik abgebaut wird (Starke 2006: 115). So hebt Palier (2007: 85 ) hervor, dass in den letzten 15 Jahren gerade in der Rentenpolitik in zahlreichen Ländern Maßnahmen ergriffen worden sind, staatliche Ausgaben zu kürzen. Fallorientierten Analysen über konkrete Reformmaßnahmen in einzelnen Ländern können wir entnehmen, dass in der Rentenpolitik in einer Reihe von Ländern in der Tat Leistungen gesenkt, Rechtsansprüche restringiert und Märkte eingeführt wurden (z.b. Pierson 1994: 15; Green-Pedersen 2001; Korpi und Palme 2003; Palier 2006, 2007). Auch quantitative Analysen über Sozialausgaben und die Verteilung von Einkommen im OECD-Bereich sehen die Retrenchment-These mittlerweile bestätigt (Allan und Scruggs 2004; Scruggs 2006), auch wenn beispielsweise die Studie von Kenworthy und Pontusson (2005: 450) gezeigt hat, dass Wohlfahrtsstaaten - entgegen der These eines Welfare State Retrenchment - in den 1990er Jahren mehr umverteilen als in den 1980er Jahren. ${ }^{1}$ Obwohl es große Diskussionen darüber gibt, wie man die ,abhängige Variable“ zu definieren hat

1 Kenworthy und Pontusson (2005: 455) berücksichtigen jedoch in ihrer Analyse nicht das ausgabenmäßig betrachtet nach wie vor umfangreichste Wohlfahrtsprogramm, die Rentenversicherung. 
(Green-Pedersen 2004), fällt auf, dass die Frage des Abbaus von Umverteilung vor allem anhand staatlicher Sozialleistungen analysiert wird.

Geht man von der Seite der abhängigen Variable zur Seite der unabhängigen, bemerkt man, dass bei der erklärenden Analyse von Retrenchment-Prozessen oftmals auf den relativen Einfluss verschiedener politischer Faktoren rekurriert wird, wobei insbesondere der Einfluss politischer Parteien und ihres Wettbewerbs sowie die Bedeutung institutioneller Veto-Punkte untersucht wird (vgl. Huber et al. 1993; Green-Pedersen 2001; Bradley et al. 2003; Kittel und Obinger 2003; Korpi und Palme 2003; Allan und Scruggs 2004; Amable et al. 2006). Ein Fixpunkt der Debatte ist dabei die Frage, inwieweit partei(-politische) Akteure einen Einfluss ausüben, und wenn ja, ob eher die linken Parteien, die christdemokratischen Parteien oder aber die Mittelklasse und die bürgerlichen Parteien das Ausmaß staatlicher Sozialausgaben, und damit das Ausmaß an Umverteilung und Solidarität, zu verantworten haben (vgl. dazu umfassend Emmenegger 2007; Starke 2006). So kommen Korpi und Palme (2003) und Bradley et al. (2003) in ihren Analysen der Bestimmungsfaktoren staatlicher Umverteilungspolitik zu dem Ergebnis, dass die Stärke linker Parteien einen maßgeblichen Einfluss auf das Ausmaß staatlicher Umverteilungspolitik ausübt und in Ländern mit starker Linker Sozialkürzungen schwierig sind. Es gibt aber auch Studien, die zeigen, dass Sozialdemokraten erfolgreicher als rechte Parteien Sozialabbau betreiben können (Ross 2000; Kitschelt 2001; Green-Pedersen 2001). Allan und Scruggs (2004) und Amable et al. (2006) kommen zu dem Befund, dass rechte Parteien stärker kürzen.

Quantitative Analysen konzentrieren sich also auf die Frage, ob Parteien einen Einfluss haben und wenn ja, welche Parteien wie stark Retrenchmentprozesse beeinflussen. Fallorientierte Studien haben demgegenüber interessante Ergebnisse hinsichtlich der Frage hervorgebracht, in welchen konkreten Ländern Parteien einen starken Einfluss auf die Restrukturierung ausüben und in welchen nicht, weil der Parteieneffekt aufgrund von Konsenszwängen mit nicht parteilichen Akteuren wie Zweite Kammern oder Gewerkschaften und Rentnerverbänden begrenzt ist. Dabei stellen die Niederlande und Dänemark Repräsentanten der ersten Gruppe und Frankreich und Deutschland Vertreter der zweiten Gruppe dar. So hat Green-Pedersen (2001) in seiner Analyse der RetrenchmentProzesse in den Niederlanden und Dänemark deutlich gemacht, dass politische Parteien die treibenden Kräfte in den Sozialkürzungen waren. Er führt dies auf die Struktur des Parteienwettbewerbs zurück. Green-Pedersen (2006) und Green-Pedersen und Lindbom (2006) haben diesen Parteieneffekt für Dänemark bestätigt. Anderson (2006) hebt den Einfluss des Parteienwettbewerbs auf Rentenreformen in den Niederlanden hervor. Studien über Frankreich und Deutschland zeigen hingegen, dass dort der Einfluss von Parteien geringer ist. So verweisen Conceição-Heldt (2006: 150) und Palier (2007: 8990) für Frankreich auf den Einfluss der Gewerkschaften und ihrer öffentlichen Protestaktionen. Für die Rentenreformen in Deutschland haben wiederum Schludi (2005) und Schulze und Jochem (2006) gezeigt, dass Parteien nur dann erfolgreich Reformen durchführen können, wenn es Ihnen gelingt, durch Tauschgeschäfte und Verhandlungen die Zustimmung des Bundesrates und der Gewerkschaften zu erheischen. Myles und Pierson (2001) und Palier (2007) konnten zudem belegen, dass die Rentensysteme in Frankreich und Deutschland für Parteien nur schwer reformierbar sind, weil umlagefinanzierte Systeme, die dort dominieren, mit den damit verbundenen Veto-Koalitionen 
Resistenz befördern (Pierson 1994). Demgegenüber gehören Dänemark und die Niederlande zu jener Gruppe von Ländern, in denen in der Rentenpolitik ein kapitalfundiertes System überwiegt (Palier 2007).

Die Fokussierung der Umverteilungsdebatte auf staatliche Sozialpolitik und den Einfluss politischer Faktoren verwundert insofern, als es mittlerweile doch eine Reihe von Studien gibt, die darauf aufmerksam machen, dass Länder, in denen die staatliche Altersvorsorge traditionell durch Einrichtungen auf betrieblicher oder tariflicher Ebene ergänzt wurde, auch in der jetzigen Umbauphase diese hybriden Systeme beibehalten (Haverland 2001; Myles und Pierson 2001; Brooks 2002; Ebbinghaus 2006). So verweisen Myles und Pierson (2001: 330) darauf, dass spät entwickelte Wohlfahrtsstaaten, in denen das Umlagesystem weit weniger entwickelt ist und die eine starke zweite und dritte Säule aufweisen - wie die Niederlande, Dänemark und Australien - es in der jetzigen Umbauphase einfacher haben, die kapitalfundierten Renten weiter auszubauen. Brooks (2002: 500, 516) hat in ihrer quantitativen Studie zu Rentenkürzungen in 57 Staaten gezeigt, dass in Ländern mit Betriebsrenten stärker gekürzt wird. Haverland (2001) legt für die Niederlande dar, dass dessen Mehr-Säulen-System zwar das Resultat kontingenter Prozesse ist, jedoch die Tarifrenten die Retrenchmentpolitik maßgeblich beeinflussen. Betriebsrenten und Tarifrenten finden also durchaus Eingang in die Forschung: Was jedoch noch fehlt, sind zum einen systematische Untersuchungen darüber, aufgrund welcher Mechanismen und unter welchen Bedingungen nicht-staatliche kollektive Modelle der Altersvorsorge wohlfahrtsstaatliche Restrukturierung beeinflussen; zum anderen wurde noch nicht analysiert, ob und wie die Tarifrenten den Parteieneffekt beeinflussen.

Fasst man die Forschung zu Retrenchmentprozessen zusammen, so gilt es das Folgende festzuhalten: (1) Retrenchment findet nun international statt. (2) Die Forschung untersucht vor allem den Einfluss politischer Parteien. (3) Auch in den für die vorliegende Analyse ausgewählten Ländern haben in der Rentenpolitik Kürzungen stattgefunden, wobei in den Niederlanden und Dänemark Parteien einen größeren Einfluss ausüben als in Deutschland und Frankreich. (4) Die Literatur konzentriert sich in ihrer Analyse der Kürzung von Umverteilungspolitik auf staatliche Sozialpolitik, also territorial organisierte Umverteilung. (5) Hinsichtlich der unabhängigen Variablen steht die Ausgestaltung der territorialen Interessenvermittlung im Vordergrund. In den folgenden Abschnitten werde ich nun jedoch zeigen, dass eine Analyse, die sowohl auf Seite der abhängigen Variable als auch Seite der unabhängigen Variablen die Arbeitsbeziehungen einbringt, eine sinnvolle Ergänzung für die Untersuchung wohlfahrtsstaatlicher Retrenchmentprozesse darstellt.

\section{Das Modell funktionaler Umverteilung durch tarifliche Sozialpolitik}

Wie bereits ausgeführt differenziert dieser Artikel in Anlehnung an Marshall zwischen zwei Modellen der Umverteilung. Umverteilung, die auf dem territorialen Prinzip beruht, und Umverteilung, die funktional organisiert ist. Thomas Marshall (1964) hat mit seinem Konzept des „Social Citizenship“ darauf verwiesen, dass wohlfahrtsstaatliche Umverteilung die sequentielle Institutionalisierung von bürgerlichen, politischen und 
sozialen Bürgerrechten (,civic citizenship“, „political citizenship“, „,social citizenship“) zur Voraussetzung hat. Mit seiner Unterscheidung zwischen politischen und industriellen Bürgerrechten (,industrial citizenship“) machte Marshall (1964: 94) darauf aufmerksam, dass aufgrund von Tarifverträgen „sozialer Fortschritt“ nicht nur durch soziale Rechte, folglich durch Gesetze zu einem Mindestlohn oder einer verpflichtenden Sozialversicherung, sondern auch durch industrielle Bürgerrechte geschaffen werden kann. Gewerkschaften, so Marshall (1964: 94), können durch Tarifverträge „ein sekundäres System von Bürgerrechten“ - eben Industrielle Bürgerrechte - etablieren, das „parallel und ergänzend zum System ,politischer Bürgerrechte“ steht.

Kollektive Formen sozialer Sicherung - nicht-marktliche Institutionen, die Umverteilung organisieren - sind also das Ergebnis des Gebrauchs von ,ppolitischen Bürgerrechten“ und ,industriellen Bürgerrechten“. Tarifliche Sozialpolitik kann als ein Umverteilungsmodell interpretiert werden, das zwar weniger Solidarität als staatliche Sozialpolitik offeriert, das aber immer noch mehr umverteilt als rein marktliche Lösungen. Gleichwohl bedeutet tarifliche Sozialpolitik auch weniger Umverteilung und Solidarität. In einem System tariflicher Sozialpolitik basiert Umverteilung nämlich nicht mehr auf dem territorialen Prinzip, in dem mit Hilfe nationaler Fonds zwischen Sektoren, Berufen und Firmen Risikoausgleich betrieben wird, sondern auf dem funktionalen Prinzip. Gemäß diesem Prinzip bestimmt in erster Linie die Produktivität der dem Tarifvertrag anhängigen Firmen den Grad der Umverteilung, was freilich nicht ausschließt - und dies zeigt insbesondere der niederländische Fall -, dass kollektivvertragliche oder rechtliche Regelungen das Umverteilungspotential erhöhen können. In funktional organisierter Umverteilung entscheidet die Zugehörigkeit zu einem Tarifvertrag über redistributive Leistungen, in territorial organisierter Umverteilung die Staatsbürgerschaft, der Wohnort oder ob der Arbeitsvertrag in den Anwendungbereich nationalen Arbeits- und Sozialrechts fällt. In einem territorialen Modell ist Umverteilung also an die Gebietsherrschaft gebunden, nicht jedoch zwangsläufig an die Nationalität. In Ländern, in denen tarifliche Sozialpolitik existent ist, wird der territorial organisierten Umverteilung und Solidarität demgegenüber ein Modell angefügt, das primär von einer funktionalen Koalition zwischen Gewerkschaften und Arbeitgebern getragen wird.

Im Folgenden soll nun geprüft werden, inwieweit es in den ausgewählten Ländern tarifliche Regelungen zur Altersvorsorge gibt, welche Tradition und welchen Entwicklungsstand diese aufweisen. Zuvor werden jedoch zunächst die Rentensysteme kurz charakterisiert.

Gemeinsames Element des dänischen und niederländischen Rentensystems ist es, dass in beiden Ländern eine relativ generöse Grundrente mit Einheitsleistungen der zentrale Baustein der ersten Säule, der staatlichen Altersvorsorge, darstellt (Anderson 2006; Green-Pedersen 2006). Das niederländische AOW-System wird aus Beiträgen auf alle Einkommen finanziert, ist nach dem Kapitalprinzip organisiert und an das Mindesteinkommen indexiert. Die dänische Grundsicherung besteht aus zwei Elementen: Zum einen aus der nach dem Umlageprinzip organisierten Folkepension, die von Steuern getragen wird, deren Bezug an Wohnsitzvoraussetzungen gebunden ist und die an Lohnentwicklung im privaten Sektor gekoppelt ist; zum anderen aus einem zusätzlichen verpflichtenden, kapitalfinanzierten Altersvorsorgesystem (ATP), das 1964 eingeführt wurde und in dem die Beiträge und Leistungen nicht nach Einkommen, sondern nach 
Arbeitszeit berechnet werden. Die Grundrenten sind in beiden Ländern für Rentner die wichtigste Rentenart (Frericks et al. 2006: 478-481). Was die beiden Länder des Weiteren verbindet ist, dass die zweite Säule umfassend ist und aus Tarifrenten, d.h. Altersvorsorgeeinrichtungen, die durch Branchentarifverträge reguliert sind, besteht. Damit wird die durch die Grundrenten bereitgestellte territoriale Umverteilung durch funktionale Umverteilung ergänzt. Während diese Tarifrenten in den Niederlanden eine lange Tradition aufweisen und der Beitritt zu sektoralen Altersvorsorgefonds für Unternehmen seit 1949 durch ein Gesetz verpflichtend ist, wurde in Dänemark die tarifliche Altersvorsorge (AMP) erst 1991 eingeführt. Bereits in den 1960er und 1970er Jahren wurden jedoch auch in Dänemark im öffentlichen Sektor Tarifverträge zur betrieblichen Altersvorsorge geschlossen (Green-Pedersen und Lindbom 2006: 252). Sowohl in Dänemark als auch in den Niederlanden existieren als drittes Element der Altersvorsorge, als dritte Säule, individuelle, private und freiwillige Sparpläne.

Gemeinsames Merkmal des französischen und deutschen Rentensystems ist, dass eine umlagefinanzierte, für alle Beschäftigte verpflichtende Altersvorsorge das Rentensystem dominiert. Das französische Rentensystem besteht schon lange Zeit aus drei Säulen. Zur ersten Säule wird in Frankreich nicht nur das umlagefinanzierte staatliche Basissystem (régime général) gezählt, sondern auch die umlagefinanzierte, durch nationale (!) Tarifverträge regulierte obligatorische berufliche Zusatzvorsorge (retraite complémentaire). Zur zweiten Säule zählt man freiwillige betriebliche kapitalgedeckte $\mathrm{Zu}-$ satzkassen (retraite supplémentaire), zur dritten Säule die private Vorsorge (Veil 2004: 53). Im Durchschnitt besitzen die Rentner aus 2,8 Systemen eine Altersvorsorge (Veil 2004: 53). Die erste und zweite Säule sind umlagefinanziert. Die seit 1972 obligatorische berufliche Zusatzvorsorge wird in der Privatwirtschaft von ARRCO (Association des Régimes de Retraite Complémentaire) verwaltet. Im deutschen Rentensystem dominierte hingegen bis vor kurzem die gesetzliche Rentenversicherung. Betriebliche Renten standen vor allem den Beschäftigten des öffentlichen Dienstes und den besser bezahlten Angestellten der Privatwirtschaft zur Verfügung. Mit dem Tarifvertrag von 1998, der in der chemischen Industrie abgeschlossen wurde, erhielt die tarifliche Altersvorsorge im verarbeitenden Gewerbe Einzug. ${ }^{2} 2001$ wurde die tarifliche Altersvorsorge mit der Riester'schen Rentenreform schließlich - neben der privaten Altersvorsorge - weiter ausgebaut. Im selben Jahre schloss auch die Metall verarbeitende Branche einen Tarifvertrag zur Altersvorsorge ab.

In Deutschland gründet die Altersvorsorge also maßgeblich auf dem territorialen Umverteilungsmodell. Dies ist auch in Frankreich der Fall, obwohl die umlagefinanzierte obligatorische berufliche Zusatzvorsorge (retraite complémentaire) auch auf nationalen, sektorübergreifenden Tarifverträgen beruht, die 1947 bzw. 1961 zum ersten Mal abgeschlossen wurde, die aber einen Gefahrenausgleich zwischen verschiedenen Sektoren beinhalten (vgl. Tab. 1). Die Teilnahme an dieser Zusatzvorsorge ist ferner seit einem Gesetz von 1972 für alle Beschäftigten und Unternehmen, die auch dem Basissystem angehören (!), verpflichtend. Das bedeutet: Entscheidend für die Zugehörigkeit zum Umverteilungsmodell der Zusatzvorsorge ist, dass der Arbeitsvertrag in den Bereich

2 Ich betrachte im deutschen Fall nur die Sektoren Chemie und Metall. In der Bauindustrie und im öffentlichen Sektor gibt es die tarifliche Altersvorsorge schon seit längerer Zeit. 
des französischen Sozial- und Arbeitsrechts fällt. Zugehörigkeit wird nicht durch den Tarifvertrag bestimmt. Die nationalen Tarifverträge sorgen vielmehr dafür, dass die Interessen der Unternehmen und Arbeitnehmer berücksichtigt werden. Sie legen Entscheidungskompetenzen der Sozialpartner in der Bestimmung von Beiträgen und Leistungen fest; dies aber eben über Unternehmen, Berufe und Sektoren hinweg (ARRCO 2001). Man kann es allgemeiner auch so formulieren: In Frankreich hat der Staat ein funktionales Umverteilungsmodell kooptiert und es so in ein territoriales umgewandelt.

Systeme der tariflichen Altersvorsorge gibt es heute also in allen vier Ländern, jedoch unterscheiden sie sich, wie die Tabelle 1 zeigt, hinsichtlich ihrer strukturellen Merkmale erheblich.

Die Niederlande haben das am besten entwickelte System der tariflichen Altersvorsorge und Deutschland das schlechteste. Das niederländische System gilt ferner als ein Prototyp für eine hochgradig solidarische tarifliche Altersvorsorge, der durch ,vier kollektive soziale Mechanismen“ - Konvention, Konvenants, Tarifvertrag und Zwang - gestützt wird (Rein und Turner 2001: 137). Auch in Dänemark und Frankreich sind die Deckungsgrade sehr hoch.

Wie ist es dazu gekommen, dass in den Niederlanden und Dänemark die funktionale Umverteilung einen relativ hohen Stellenwert einnimmt, während die Altersvorsorge in Frankreich und Deutschland auf dem territorialen Prinzip beruht? Bevor ich im Folgenden darlege, dass der unterschiedliche Mix an territorial und funktional organisierter Umverteilung mit Prozessen der Institutionalisierung der Arbeitsbeziehungen und Verläufen der Demokratisierung in einen Zusammenhang gebracht werden kann, erläutere ich nun zunächst die in diesem Artikel angewendete Vergleichsmethode.

\section{Die Methode: Die Verknüpfung von Ländervergleich mit Prozessanalyse}

Ich kombiniere in diesem Artikel in einem zweistufigen Vergleich die Mill'schen Methoden der Differenz und Konkordanz, ohne mich dabei jedoch dem mit diesen Methoden verbundenen deterministischen Kausalitätsbegriff anzuschließen. Im ersten Vergleich, dem historischen Teil der Untersuchung, untersuche ich die institutionellen Bedingungen von funktionalen Umverteilungseinrichtungen (Tarifrenten). Im zweiten Vergleich, dem Vergleich über die aktuelle wohlfahrtsstaatliche Prozesspolitik, argumentiere ich, dass das Ausmaß funktionaler Umverteilung den Parteieneffekt in der Phase des Retrenchments beeinflusst. Diese beiden Ländervergleiche werden durch fallinterne Analysen der ausgewählten Fälle ergänzt. Die fallinternen Analysen dienen mir dazu, meine Untersuchung im Stile eines process tracing historisch zu kontextualisieren. Damit ist auch klar, dass das Ziel meiner vergleichenden Analyse nicht der Nachweis von kausalen Effekten ist, sondern die Identifikation von Mechanismen. Ich will nicht deduzieren und falsifizieren, sondern ich gehe explorativ, heuristisch und induktiv vor.

Die Konkordanz- und die Differenzmethode gehen auf John Stuart Mill (1874) zurück. Verwendet man die Methoden kausalanalytisch, so können mit der Differenzmethode Unterschiede in der „abhängigen“ Variablen mit Unterschieden in möglichen „unabhängigen“ Variablen erklärt werden; die Konkordanzmethode wird demgegenüber angewandt, um Gemeinsamkeiten in der „abhängigen Variable“ mit Gemeinsamkeiten 


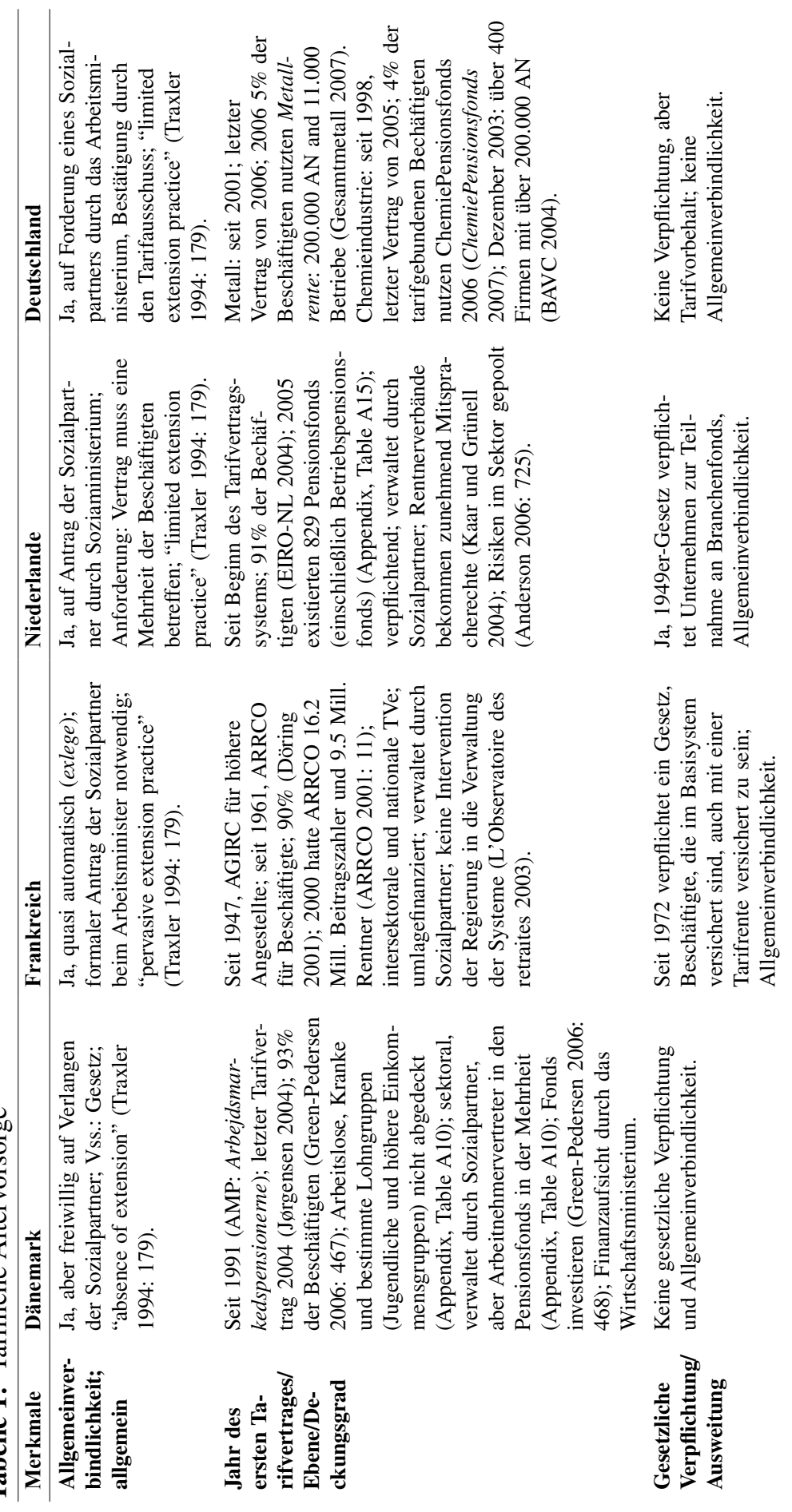




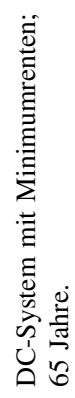

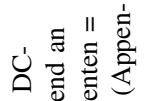

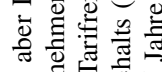

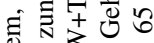

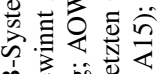

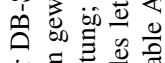

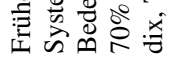

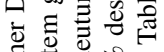

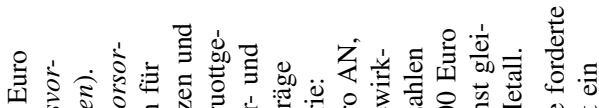

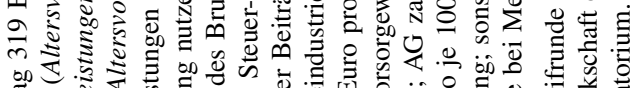

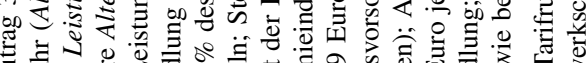

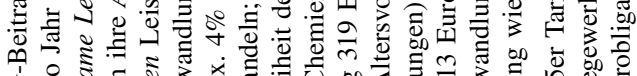

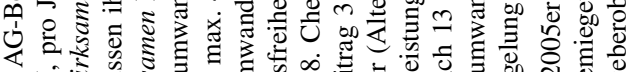

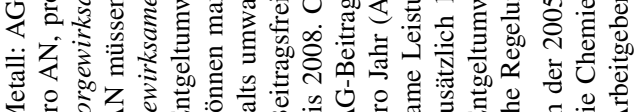

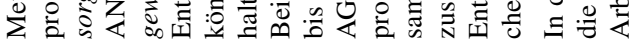

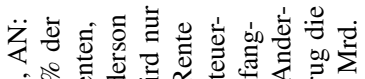

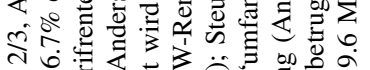
तิ

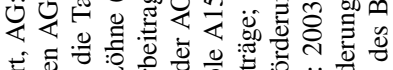

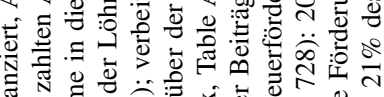

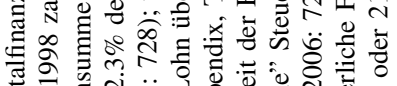

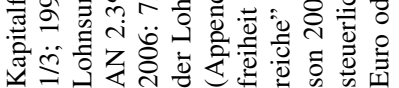

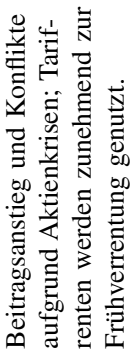

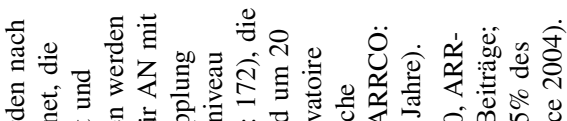

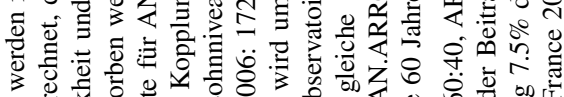

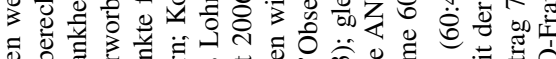

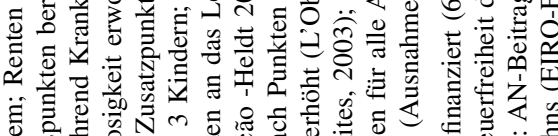

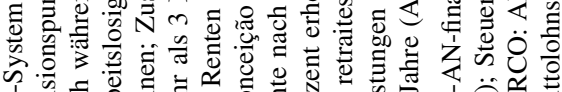

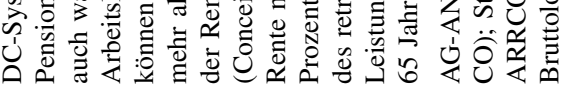

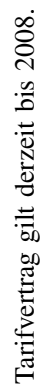
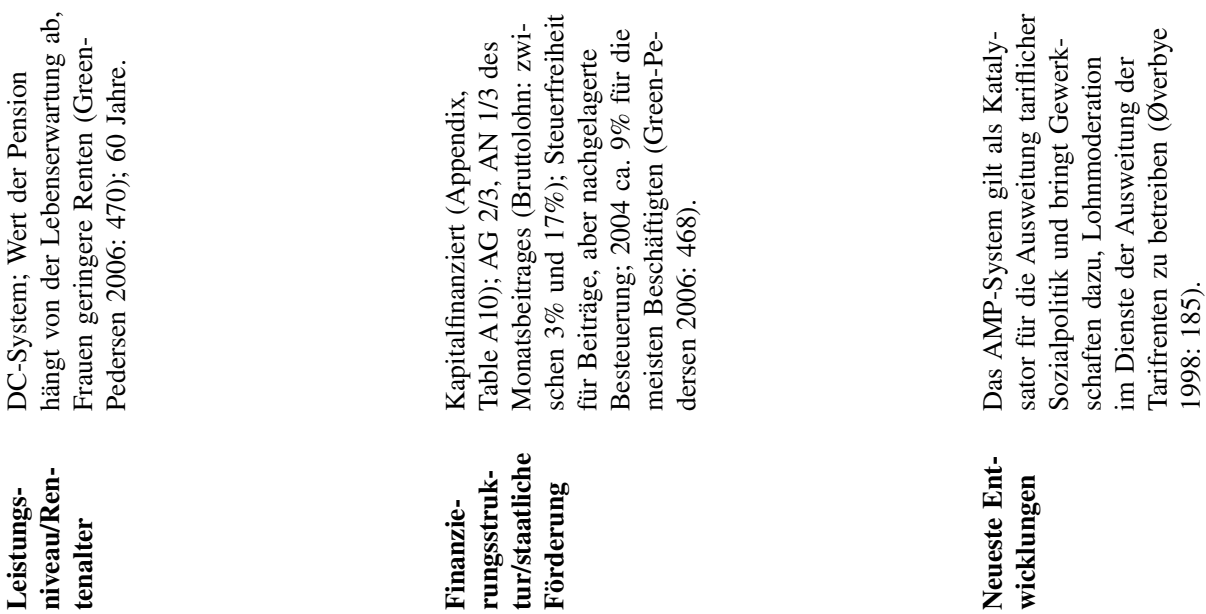

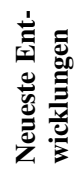


in „unabhängigen Variablen“ zu erklären. Dabei ist zu berücksichtigen, dass Mill seinen Methodenkanon auf der Grundlage eines deterministischen, und damit auch statischen Kausalitätsbegriff formuliert hat. Während die Differenzmethode hinreichende Bedingungen eines bestimmten Phänomens zu identifizieren sucht, trägt die Konkordanzmethode zur Bestimmung von notwendigen Bedingungen bei. Die Differenz- und Konkordanzmethode können auch kombiniert angewandt werden, worauf nicht nur Mill (1874: 283-284) selbst verwiesen hat („Indirect method of difference“ bzw. „Joint method of agreement and difference"), sondern auch Ragin (1987: 39-42) und Skocpol und Somers (1980: 183). Aufgrund ihres deterministischen Verständnisses von Kausalität setzen die Mill'schen Methoden die Abwesenheit von Messfehlern und Interaktionseffekten sowie Monokausalität voraus (Lieberson 1991). Über die damit verbundenen Vor- und Nachteile wie auch generell über die Annahmen und die Zweckmäßigkeit der Mill'schen Methoden wird in der vergleichenden Politikwissenschaft kontrovers diskutiert (Jahn 2007: 17-18; Lieberson 1991).

Prozessanalyse findet in der vergleichenden Politikwissenschaft zunehmend Berücksichtigung. Dabei wird sie auch als eine Methode der fallinternen Analyse verwendet, die qualitative oder quantitative Vergleichsstudien sinnvoll ergänzt. Das Ziel von Prozessanalysen ist es, Entwicklungsprozesse zu rekonstruieren. Dadurch können Scheinkorrelationen oder Äquifinalitäten (mehrere Bedingungen führen zu einem Ergebnis) aufgedeckt werden, kann der Einfluss von intervenierenden Variablen auf Zusammenhänge zwischen unabhängigen und abhängigen Variablen genauer untersucht werden oder wird über sequentielle oder situative Interaktionseffekte aufgeklärt (Mahoney 2004: 88-90; Blatter et al. 2007: 157-166). Die Prozessanalyse, die mittlerweile zahlreiche Akzentuierungen erfahren hat (George und Bennett 2005: 205-232; Blatter et al. 2007: 157-166), trägt durch Kontextualisierung der Evidenzen in Raum und Zeit zu mehr Tiefenschärfe bei. In Prozessanalysen steht damit weniger die Frage eines kausalen Effektes im Vordergrund als die Identifikation von kausalen Mechanismen. Es geht hier also nicht um die Identifikation der Wirkung, die sich bei einer abhängigen Variable aufgrund der Änderungen einer unabhängigen Variable feststellen lässt, sondern um die Spezifikation derjenigen ,wiederkehrenden Prozesse, die bestimmte Ursachen mit bestimmten Wirkungen verbinden" (Mayntz 2002: 24).

Wie setze ich in meinem Vergleich nun die Verknüpfung des Ländervergleiches mit Prozessanalyse konkret um? Meine Analyse betont die Bedeutung von Entwicklungspfaden. Konkret will ich zeigen, dass Muster der Institutionalisierung der Arbeitsbeziehungen und der Demokratisierung aufgrund der damit verbundenen Schaffung von Einrichtungen der funktionalen Umverteilung historische Weichensteller für aktuelle politische Reformpräferenzen sein können und den Parteieneffekt in der jetzigen wohlfahrtsstaatlichen Restrukturierung beeinflussen. Die Analyse will keine rivalisierenden Erklärungen ausschließen, sondern zielt vielmehr auf eine konfigurative Erklärung, die die Bedeutung von historischen Abfolgen plausibel machen soll. Dementsprechend richtet sich meine Fallauswahl weniger danach, bestimmte erklärende Faktoren zu kontrollieren. Die Auswahl der Fälle erfolgt vielmehr theorieorientiert und - bewusst - nach der abhängigen Variable: theorieorientiert, insofern die ausgewählten Fälle für jeweils zwei spezifische Konfigurationen der Institutionalisierung der Arbeitsbeziehungen und der Verläufe der Demokratisierung stehen, die es erlauben, Marshalls Differenzierung 
zwischen politischen und industriellen Staatsbürgerrechten für die Analyse von Parteieneffekten zu nutzen und weiter zu entwickeln; nach der ,abhängigen Variable“, weil die Fälle danach ausgewählt wurden, ob sie eine Tradition funktionaler Umverteilung aufweisen (Tarifrenten) und sich in der gegenwärtigen Phase der Restrukturierung ein Parteieneffekt zeigt. Dabei ist gleichwohl anzumerken, dass eine Fallauswahl nach der abhängigen Variable in der vergleichenden Politikwissenschaft sehr umstritten ist, weil sie mit dem Problem des selection bias verbunden ist (Ebbinghaus 2005). ${ }^{3}$

Abb. 1 erläutert mein Vorgehen im zweistufigen Ländervergleich. Im Vergleich zwischen den beiden Ländergruppen Dänemark und Niederlande auf der einen und Deutschland und Frankreich auf der anderen Seite gelangt in beiden Stufen die Differenzmethode zur Anwendung. Im Vergleich zwischen Dänemark und den Niederlanden und zwischen Frankreich und Deutschland wende ich jeweils die Konkordanzmethode an. In Anlehnung an Skocpol (1979: 37) verwende ich meine Fälle als gegenseitige konfigurative Kontrastfolien. Das heisst, ich habe zwei Gruppen von Fällen ausgewählt, wobei in der einen Gruppe das Phänomen, das man verstehen will (Tarifrenten, Parteieneffekt), auftritt (positive cases, Skocpol 1979: 37), und in der anderen nicht (negative cases, Skocpol 1979: 37). Die Logik meines Vergleiches besteht dementsprechend darin, innerhalb der positiven Fälle der Konkordanzmethode entsprechend nach Gemeinsamkeiten in den unabhängigen Variablen zu suchen. Diese positiven Fälle konfrontiere ich dann mit der negativen Gruppe, womit die Differenzmethode zur Anwendung gelangt. In den negativen Fällen prüfe ich zudem - wiederum anhand der Konkordanzmethode -, ob in diesen die in den positiven Fällen gefundenen unabhängigen Variablen nicht auftreten. Auch die Prozessanalyse wende ich in zweifacher Hinsicht an. Zum ersten ist meine Analyse in ihrer generellen Anlage prozessanalytisch, insofern ich meine Länder über einen längeren Zeitraum betrachte und einen zweistufigen Vergleich - historischer Vergleich und Vergleich der aktuellen Prozesspolitik - vornehme. Zum zweiten wende ich Prozessanalyse im ersten Vergleich, dem historischen Vergleich, an, um die Bedeutung von Sequenzen der Demokratisierung und Institutionalisierung der Arbeitsbeziehungen in der Entstehung von Tarifrenten aufzuzeigen. Zusammenfassend gilt es somit festzuhalten: Meine Untersuchung ergänzt einen qualitativen Ländervergleich mit fallinterner Prozessanalyse, um so den Faktor „Zeit“ durch eine historische Kontextualisierung und Längsschnittbetrachtung in die Analyse zu integrieren.

\section{Institutionelle Bedingungen tariflicher Sozialpolitik}

Tarifverträge können soziale Leistungen bereitstellen. Daran schließt sich die Frage an, unter welchen Bedingungen Gewerkschaften und Arbeitgeberverbände ein System tariflicher Sozialpolitik etablieren. Unter Bezugnahme auf Marshalls Differenzierung zwischen politischen und industriellen Bürgerrechten lassen sich diesbezüglich die fol-

3 Jedoch schließe ich mich hier Ebbinghaus (2005: 144-145) an, der argumentiert, dass eine Auswahl nach der abhängigen Variable so lange kein Problem darstellt, als man nicht über die Fälle hinaus generalisieren will, sondern im Gegenteil die Fälle als Konfigurationen behandelt, die komplexe Entwicklungspfade repräsentieren. 
Abbildung 1: Die Verknüpfung des Ländervergleiches mit Prozessanalyse

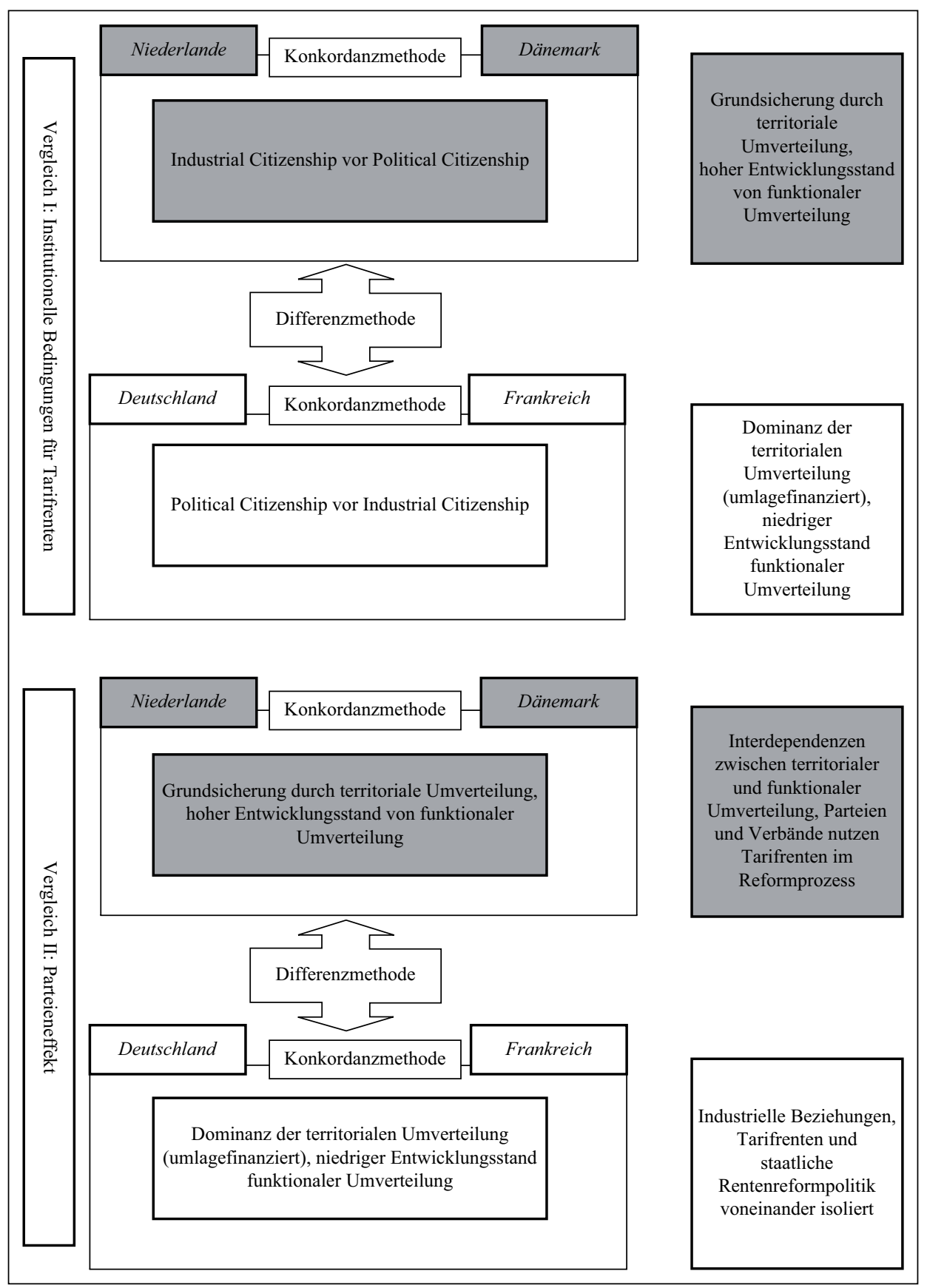


genden beiden Thesen formulieren: (1) Die Sequenz der Institutionalisierung von politischen und industriellen Bürgerrechten beeinflusst das Ausmaß tariflicher Sozialpolitik entscheidend (Ebbinghaus 1995: 66). (2) Gesellschaften, in denen industrielle Bürgerrechte vor politischen Bürgerrechten institutionalisiert und genutzt wurden, weisen ein höheres Ausmaß an funktional organisierter Umverteilung auf als Länder, in denen die Sequenz umgekehrt verlief. Soziale Rechte können also unterschiedlich institutionalisiert werden und die industriellen Beziehungen dürfen nicht vernachlässigt werden, wenn man den sozialpolitischen Entwicklungsstand von Gesellschaften analysiert.

Ebbinghaus (1995: 56) zufolge erfüllen politische und industrielle Staatsbürgerrechte unterschiedliche Funktionen und mobilisieren in unterschiedlichen Arenen. Ob Verbände den politischen Raum oder den wirtschaftlichen Raum nutzen, wird maßgeblich durch historische Institutionalisierungsprozesse beeinflusst, insbesondere von Pfaden der Integration von Gewerkschaften und Arbeitgebern in Politik und Ökonomie im Verlauf der Industrialisierung, des nationbuilding und der Staatsentwicklung. Wenn die politischen Bürgerrechte früher entwickelt waren als die industriellen Bürgerrechte, haben die Verbände den politischen Raum genutzt, um ihre sozialpolitischen Forderungen umzusetzen. Dies hat die Entwicklung von staatlicher Sozialversicherung befördert und von tariflicher Sozialpolitik verzögert. Wenn hingegen die industriellen Bürgerrechte früher entwickelt waren als die politischen und die Verbände die Tarifpolitik auch umfassend für die Regulierung des Arbeitsmarktes genutzt haben, so hat dies die Entwicklung staatlicher Sozialgesetzgebung gebremst. Dies hat zum Beispiel dazu führt, dass Tarifverträge in der sozialen Sicherung eine zentrale Funktion übernehmen, oder anders formuliert, territorial organisierte Umverteilung mit funktional organisierten Umverteilungsleistungen ergänzt wird. Mit anderen Worten: Mit Bezug auf Marshall und Ebbinghaus argumentiere ich, dass die Sequenz der Institutionalisierung von politischen und industriellen Staatsbürgerrechten Opportunitätsstrukturen festlegt, die die sozialpolitischen Präferenzen von Staat und Verbänden maßgeblich beeinflussen. Im Folgenden soll die Plausibilität dieser Hypothesenkette anhand der ausgewählten Länder mit den Mill'schen Methoden der Differenz und Konkordanz illustriert werden.

Aus der vergleichenden Forschung zu Gewerkschaften, Arbeitsbeziehungen und Wohlfahrtsstaaten kann man eine Reihe von Indikatoren destillieren, anhand derer man die Sequenz der politischen und wirtschaftlichen Integration operationalisieren kann (z. B. Armingeon 1994; Ebbinghaus 1995).

Die zeitliche Abfolge der Institutionalisierung von politischer und wirtschaftlicher Integration kann man anhand der Einführungsjahre der folgenden Rechte „messen“ (Ebbinghaus 1995): Hinsichtlich der politischen Integration ist die Einführung von Versammlungsfreiheit, das erste Jahr, in dem mindestens 50\% der männlichen Bevölkerung wahlberechtigt war, die Einführung einer gegenüber dem Parlament verantwortlichen Regierung und die Einführung des Verhältniswahlrechts ausschlaggebend. Für die wirtschaftliche Integration sind die folgenden Rechte maßgebend: die Einführung der Koalitionsfreiheit, des Streikrechts, das Jahr des Abschlusses des ersten wichtigen nationalen Tarifvertrages und die Einführung von Mitbestimmungsrechten. Als einen weiteren Indikator für die Frage der zeitlichen Abfolge der politischen und wirtschaftlichen Integration führe ich ferner den Anteil der Wahlbeteiligung im Jahr der Einführung 


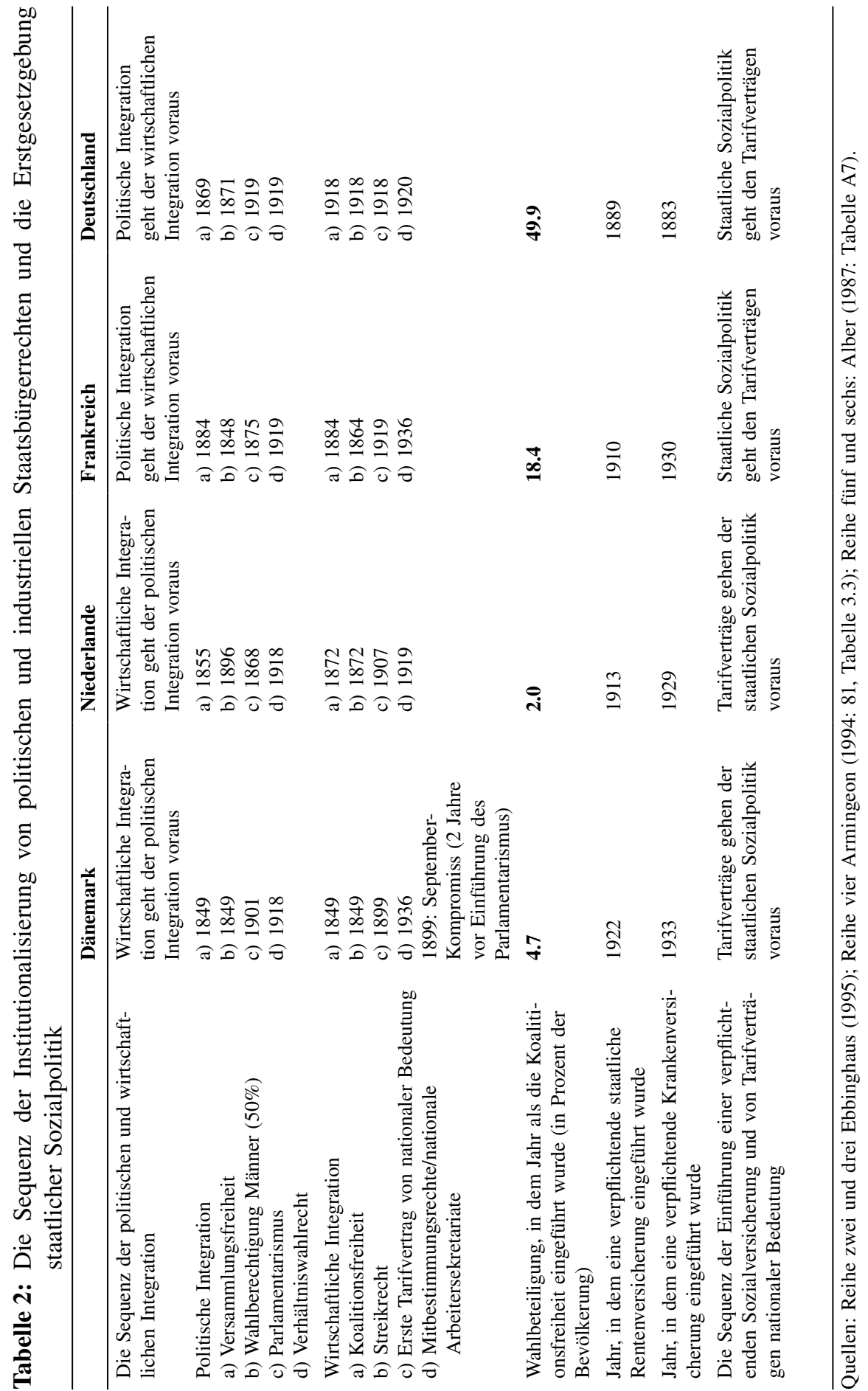


der Koalitionsfreiheit an. Ist diese hoch, so erfolgte die politische Integration vor der wirtschaftlichen Integration.

Vergleicht man nun die Niederlande, Dänemark, Frankreich und Deutschland hinsichtlich der Sequenz der Institutionalisierung und Nutzung von politischen und industriellen Bürgerrechten, wird die folgende These plausibel: Das in den Niederlanden und Dänemark stärkere Ausmaß an funktionaler Umverteilung steht damit in einem Zusammenhang, dass in diesen beiden Ländern die Schaffung und Nutzung industrieller Bürgerrechte der der politischen Bürgerrechte vorausging, während in Deutschland und Frankreich zunächst die politischen Bürgerrechte genutzt wurden (vgl. Tab. 2). Unter Anwendung der Differenzmethode kann man also das unterschiedliche Ausmaß an funktionaler Umverteilung in Dänemark und den Niederlanden auf der einen Seite sowie in Frankreich und Deutschland auf der anderen Seite mit unterschiedlichen Verläufen der Demokratisierung und der Instititutionalisierung der Arbeitsbeziehungen in einen Zusammenhang bringen.

Dänemark und die Niederlande, die beide ein entwickeltes System an sektoral organisierter, funktionaler Altersvorsorge aufweisen, sind bezüglich der ,unabhängigen Variable“ ähnlich, so dass mit Hilfe der Konkordanzmethode die Plausibilität der eben formulierten These weiter bestärkt werden kann: Der erste nationale Tarifvertrag wurde in Dänemark 1899 geschlossen, während eine dem Parlament verantwortliche Regierung erst 1901 eingeführt wurde. Demgegenüber wurde in Dänemark die Koalitionsfreiheit bereits 1849 eingeführt, wobei in diesem Jahr jedoch erst 4,7 Prozent der Bevölkerung an Wahlen teilnahmen. Wie in Dänemark ging in den Niederlanden die Institutionalisierung der wirtschaftlichen Integration der politischen voraus. Der erste nationale Tarifvertrag wurde in den Niederlanden 1907 geschlossen, das Verhältniswahlrecht jedoch erst 1918 eingeführt. Als 1872 die Koalitionsfreiheit eingeführt wurde, nahmen nur 2,0 Prozent der Bevölkerung an Wahlen teil. Eine weitere Gemeinsamkeit zwischen diesen beiden Ländern besteht ferner darin, dass die Einrichtung einer staatlichen Rentenversicherung erst nach dem Abschluss von ersten Tarifverträgen mit nationaler Bedeutung erfolgte: So wurde in Dänemark 1922 und in den Niederlanden 1913 eine staatliche Rentenversicherung eingeführt, der Abschluss von Tarifverträgen mit nationaler Bedeutung erfolgte hingegen in Dänemark bereits 1899 und in den Niederlanden 1907.

Auch Deutschland und Frankreich, die beide ein weniger gut entwickeltes System an sektoral organisierter Altersvorsorge aufweisen, zeigen in der „unabhängigen Variable“ eine Ähnlichkeit auf, so dass auch in dieser Ländergruppe die Konkordanzmethode zur Anwendung kommen kann: In beiden ging die politische Integration der wirtschaftlichen voraus. So wurde in Frankreich der erste nationale Tarifvertrag erst 1919 geschlossen, und damit 44 Jahre nachdem die parlamentarische Regierungsform begründet wurde. Als 1884 die Koalitionsfreiheit eingeführt wurde, nahmen bereits 18,4 Prozent der Bevölkerung an Wahlen teil. Auch in Deutschland ging die politische Integration der wirtschaftlichen voraus. Der erste nationale Tarifvertrag wurde 1918 geschlossen, aber bereits 1871 waren 50 Prozent der männlichen Bevölkerung wahlberechtigt. Als 1918 die Koalitionsfreiheit eingeführt wurde, nahmen 49,9 Prozent der Bevölkerung an Wahlen teil. Eine weitere Gemeinsamkeit zwischen diesen beiden Ländern besteht ferner darin, dass die Einrichtung einer staatlichen Rentenversicherung - im Gegensatz zu den Niederlanden und Dänemark - erst nach dem Abschluss von ersten Tarifverträgen 
mit nationaler Bedeutung erfolgte: In Frankreich und Deutschland wurde die staatliche Rentenversicherung 1910 bzw. 1889 eingeführt, zu ersten nationalen Tarifverträgen von Bedeutung kam es jedoch erst 1919 und 1918.

\section{Die prozesspolitische Bedeutung tariflicher Sozialpolitik auf den Effekt von Parteien}

Basierend auf Marshalls Unterscheidung von politischen und industriellen Staatsbürgerrechten wurden eben konzeptionelle Überlegungen formuliert, die erlauben, tarifliche Sozialpolitik - und damit funktional definierte Umverteilung -, in die Analyse wohlfahrtsstaatlicher Restrukturierungsprozesse miteinzubeziehen. Die unterschiedliche Entwicklung der tariflichen Altersvorsorge und funktionaler Umverteilung in den untersuchten Ländern koinzidiert mit unterschiedlichen Pfaden der Formierung der politischen und wirtschaftlichen Integration. Während in den Niederlanden und Dänemark, wo das territoriale Modell nur eine Grundsicherung mit Einheitsleistungen bietet, industrielle Bürgerrechte vor politischen Bürgerrechten institutionalisiert und genutzt wurden, war dies in Frankreich und Deutschland, wo das territoriale Modell umfassend organisiert ist und das Umlageverfahren dominiert, genau umgekehrt. Im Folgenden soll nun als eine zweite These geprüft werden, ob historische Institutionalisierungsprozesse des politischen und des wirtschaftlichen Raumes auch in der Rückbauphase des Wohlfahrtsstaates Prozesse der Präferenzformierung hinsichtlich des Public-Private-Mix maßgeblich beeinflussen und so einen Erklärungsbeitrag dazu leisten, warum der Parteieneffekt in den Niederlanden und Dänemark grösser ist als in Deutschland und Frankreich.

Wie bereits ausgeführt, weisen Fallstudien darauf hin, dass der Einfluss politischer Parteien auf rentenpolitische Reformen in Dänemark und den Niederlanden groß ist, während die Parteien in Frankreich und Deutschland starken Konsenszwängen unterworfen sind, die ihren Einfluss mindern. Aufbauend auf die unterschiedlichen Verläufe der Institutionalisierung der Arbeitsbeziehungen und der Demokratisierung in den vier Ländern - wobei sich Dänemark und die Niederlande sowie Deutschland und Frankreich ähneln - möchte ich nun zeigen, dass in Dänemark und den Niederlanden die Präferenzformierung von Staat und Verbänden hinsichtlich des Public-Private-Mix in der sozialen Sicherung anderes verlaufen ist als in Frankreich und Deutschland. In den Niederlanden und Dänemark definieren Staat und Verbände tarifliche Altersvorsorge als komplementär zur staatlichen Altersvorsorge. In Deutschland und Frankreich haben die Sozialpartner demgegenüber lange Zeit der territorialen Altersvorsorge den Vorzug gegeben. Dies führt nicht nur dazu, dass sie rentenpolitischen Kürzungsvorschlägen seitens der Parteien einen immensen Widerstand entgegensetzen, zumal da umlagefinanzierte Systeme generell mit der Bildung von Veto-Koalitionen einher gehen. Konsequenz ist auch, dass sich die Systeme der tariflichen Altersvorsorge auf prozesspolitischer Ebene als relativ isoliert von Reformen der staatlichen Rentenpolitik erweisen.

Vor allem für die 1980er und 1990er Jahre weisen eine Reihe von Studien für die Niederlande darauf hin, dass die tarifliche Sozialpolitik - und darunter gerade auch die tarifliche Altersvorsorge - wohlfahrtsstaatliche Restrukturierungsprozesse in den Niederlanden beeinflusst hat (Haverland 2001; Trampusch 2005). So haben die Tarif- 
partner, als die niederländische Regierung Mitte der 1980er Jahre damit begann, umfangreiche Kürzungsmaßnahmen in den sozialen Sicherungssystemen einzuleiten, das Tarifvertragssystem vermehrt für soziale Leistungen geöffnet (Trampusch 2005). Zum einen wurden neue Leistungen eingeführt, wie die Tarifverträge zur Frühverrentung, zum anderen wurden Kürzungen in den staatlichen Leistungen durch eine Ausweitung der tariflichen Leistungen „kompensiert“. Letzteres ließ sich vor allem in den Tarifverträgen zur Altersvorsorge beobachten (Caminada und Goudswaard 2005: 175-176). Die tarifpolitische Abteilung des niederländischen Sozialministeriums konstatierte so auch bereits Ende der 1980er Jahre, ,dass die Folgen der Senkung der gesetzlichen Leistungen ... zum großen Teil durch die Tarifverträge aufgefangen wurden" (DCA 1989: 27). In den 1990er Jahren wurde die tarifliche Sozialpolitik Gegenstand dreiseitiger Absprachen, die die Reform des Wohlfahrtsstaates betreffen (Trampusch 2005). Cox (2001: 485) hat diese Indienstnahme der Tarifpolitik für die Reform der staatlichen Sozialpolitik jüngst in den Kontext der gewerkschaftlichen Lohnzurückhaltung gestellt. Er verweist ferner darauf, dass die Ausweitung tarifpolitischer Sozialpolitik, die aus Lohnzuwächsen finanziert wird, auch Ergebnis strategischer Überlegungen der Regierung ist: „Wage crowding and nonwage compensation are two mechanisms Dutch policymakers used to substantially alter labor-market relations. The brilliance of the strategy is that wage restraint, an idea once used to legitimate the expansion of the Dutch welfare state, is now used to justify dramatic curtailments of social protection" (Cox 2001: 485). Diese Kompensation für Kürzungen im staatlichen Rentensystem ist jedoch nicht nur auf die Bereitschaft der Tarifpartner zurückzuführen. Haverlands (2001) Analyse der rentenpolitischen Reformen seit den 1980er Jahren hat vielmehr deutlich gemacht, dass das niederländische Sozialrecht diese Politik insofern erheblich begünstigt hat, als im Gesetz festgelegt ist, dass die erste und zweite Säule sich zusammen zu 70 Prozent Rentenleistung summieren sollen.

Wie in den Niederlanden so zeigt sich auch in Dänemark, dass politische Parteien in rentenpolitischen Reformen auf die Tarifpolitik zurückgreifen können, was ihr „Können“" in der Rentenpolitik erheblich ausweitet. Der dänische Fall weist darüber hinaus jedoch vier Besonderheiten auf: (1) Die Einführung der tariflichen Altersvorsorge (AMP-System) war das Ergebnis einer Kumulation kontingenter Ereignisse, mittlerweile verbinden jedoch vor allem die Gewerkschaften einen strategischen Nutzen mit ihr (Green-Pedersen 2006: 479-84, Green-Pedersen und Lindbom 2006: 253-254). Während in den Niederlanden also die Kopplung von staatlicher Rentenpolitik mit Tarifrenten durch Recht und Gesetz, und damit den Staat, eingefasst wird, ist sie in Dänemark eher informeller und kontingenter Natur. (2) Kurz nach der Einführung der tariflichen Altersvorsorgesysteme verschärfte die Regierung in der staatlichen Basisaltersvorsorge die Bedürftigkeitsprüfung (Green-Pedersen 2006: 488). (3) Seit diesen Kürzungen sind in den letzten Jahren in der dänischen Rentenpolitik jedoch relativ wenig umfassende Kürzungsmaßnahmen erfolgt (Green-Pedersen 2006; Green-Pedersen und Lindbom 2006); vielmehr hat die tarifliche Altersvorsorge das dänische Rentensystem auf eine solidarischere Grundlage gestellt. (4) Die Entwicklung der tariflichen Rentenpolitik ist ferner wie in den Niederlanden an die Lohnpolitik gekoppelt (Trampusch 2007). Auch wenn also in Dänemark die Ausweitung tariflicher Altersvorsorge zu Beginn der 1990er Jahre keinem politischen Masterplan entsprungen ist, lässt sich doch sagen, dass die 
Tarifpolitik und die Möglichkeit der Devolution von Rentenpolitik in die Arbeitsbeziehungen den Reformprozess maßgeblich beeinflusst haben. Es ist desweiteren bemerkenswert, dass politische und tarifpolitische Akteure in den tariflichen Systemen ex post zunehmend einen organisations- und reformpolitischen Nutzen erkennen.

Wie in den Niederlanden und Dänemark so ist auch in Frankreich die tarifliche Altersvorsorge fest etabliert, sie ist jedoch dem territorialen Prinzip der Umverteilung zugehörig. In Frankreich lässt sich auch keine strategische Koordinierung von tariflicher und staatlicher Rentenpolitik beobachten. Im Gegensatz zu den Niederlanden sind die staatliche und die tarifliche Rentenpolitik auf prozesspolitischer Ebene sehr stark von einander separiert (Conceição-Heldt 2006). Dies hat zur Folge, dass die Regierung Reformen nur im Basissystem und im öffentlichen Sektor durchführen kann (Veil 2005: 26), wobei sie hier aber generell mit erheblichen Widerstand und Mobilisierungspotential seitens der Gewerkschaften konfrontiert ist (Conceição-Heldt 2006: 190-193). Gleichwohl ist hervorzuheben, dass die Regierung die Tarifrentensysteme seit Beginn der 1990er Jahren durch verschiedene Reformen gefördert hat, indem sie die staatliche Förderung dieser Systeme verbesserte (Conceição-Heldt 2006: 190-193). Trotzdem zeigt sich in Frankreich auf prozesspolitischer Ebene keine direkte Querverbindung zwischen der staatlichen Reformpolitik und den industriellen Beziehungen.

Für die deutsche Rentenpolitik haben zahlreiche Studien immer wieder auf das hohe Veto-Potential der Gewerkschaften und auf die prozesspolitische Isolierung zwischen staatlicher Rentenpolitik und den Arbeitsbeziehungen verwiesen (Hemerijck und Manow 2001; Schludi 2005). Jedoch zeigt sich seit der letzten Rentenreform eine Veränderung: Tarifliche Altersvorsorge wurde in Deutschland im Rahmen der Riester'schen Rentenreform 2000/2001 eingeführt. Damit wurde sie parallel zu Kürzungen in der staatlichen Rentenversicherung und zu einer Ausweitung marktförmig organisierter Altersvorsorge gegründet. Während des Reformprozesses trat dabei die Chemiegewerkschaft sehr stark als Befürworterin einer staatlichen Förderung von tariflicher Entgeltumwandlung auf. Warum jedoch die Regierung aktiv die von der Chemiegewerkschaft angebotene Option aufgriff, lässt sich auf vorrangig zwei Faktoren zurückführen: Zum einen hatte der spätere Bundesarbeitsminister Walter Riester Mitte der 1990er Jahre in seiner damaligen Funktion als Zweiter Vorsitzender der IG Metall mit seiner Idee zur Gründung von „Tariffonds“ zur Finanzierung der Frühverrentung erhebliche Vorarbeit geleistet, weil er sich damit als erster Vertreter des Führungszirkels der IG Metall für tarifliche Sozialpolitik aussprach. Zum anderen entwickelte sich im Laufe der Riesterreform und der Tarifrunden 2000 und 2001 eine tarifpolitische Dynamik, die die IG Metall aufgrund früher Abschlüsse der Chemiegewerkschaft unter Druck setzte, auch ihre Tarifverträge für die Altersteilzeit und die Entgeltumwandlung zu öffnen.

Unter Anwendung der Kombination aus Differenz- und Konkordanzmethode lässt sich somit das Folgende zusammenfassen: In den Niederlanden und Dänemark zeigen sich auf prozesspolitischer Ebene Interdependenzen zwischen funktionaler und territorialer Umverteilung. Die politischen Parteien und die Sozialpartner nutzen die Tarifpolitik in einer Zeit des Abbaus territorialer Umverteilungspolitik als Ressource für funktionale Umverteilung. In Deutschland und Frankreich sind die industriellen Beziehungen von Reformen in der staatlichen Rentenpolitik demgegenüber sehr isoliert. In den Niederlanden und Dänemark wurden darüber hinaus die industriellen Bürgerrechte vor den 
politischen Bürgerrechten genutzt, während sich in Frankreich und Deutschland das gegenteilige Muster zeigt. Auf Grundlage der vergleichenden Methode kann daher geschlussfolgert werden, dass die unterschiedlichen Verläufe der Institutionalisierung der Arbeitsbeziehungen und der Demokratisierung in den zwei Ländergruppen bedingen, dass in Dänemark und den Niederlanden der Handlungsspielraum politischer Parteien in der Reform territorialer Umverteilungspolitik durch die Tarifpolitik erweitert wird, nicht jedoch in Frankreich und Deutschland. Während in Frankreich und Deutschland die Präferenzformierung von Staat und Verbänden im Public-Private-Mix dahin gehend verlaufen ist, dass die Sozialpartner der territorialen Umverteilung gegenüber der funktionalen den Vorzug geben und dementsprechend in der Reform der staatlichen Rentenpolitik als gefestigte Veto-Akteure agieren, räumen in den Niederlanden und Dänemark die Sozialpartner der funktionalen Umverteilung gegenüber der territorialen Umverteilung einen gleichrangigen Stellenwert ein. Man kann es auch anders formulieren: Weil die politischen Parteien in Dänemark und den Niederlanden die Tarifpolitik als Flexibilitätsressource in der Restrukturierung staatlicher Rentenpolitik nutzen können, ist ihr Handlungsspielraum in der Rentenpolitik wesentlich größer als in Deutschland und Frankreich.

\section{Schlussbemerkung}

Vor dem analytischen Hintergrund der mit Marshall gemachten Unterscheidung zwischen territorialer und funktionaler Umverteilung hat dieser Beitrag auf Grundlage eines fallorientierten Vergleiches zwischen den Niederlanden, Dänemark, Frankreich und Deutschland gezeigt, dass der Entwicklungsstand funktionaler Umverteilung, also tariflicher Altersvorsorge, die Stärke von Parteieneffekten auf den Abbau staatlicher Umverteilungspolitik erklärt. Ausgangspunkt dieser Analyse waren die folgenden vier Überlegungen: (1) Staat und Verbände können mit tariflicher Sozialpolitik territorial organisierte Umverteilung, die durch staatliche Mindestleistungen und verpflichtende Sozialversicherungen erfolgt, auf funktionaler Ebene ergänzen, weshalb es sinnvoll ist, Überlegungen dahin gehend anzustellen, welches Ausmaß an Umverteilung Tarifverträge anbieten können. (2) Inwieweit Sozialpolitik auch durch Tarifverträge organisiert und finanziert wird - anders formuliert: der Mix aus territorialer und funktionaler Umverteilung - wird maßgeblich durch Verläufe der Demokratisierung und der Institutionalisierung der Arbeitsbeziehungen beeinflusst. (3) Sowohl hinsichtlich der abhängigen Variable als auch bezogen auf die unabhängige Variable sollten daher Studien, die versuchen, den Grad der Umverteilung zu erklären, die Arbeitsbeziehungen in ihre Analyse integrieren. (4) Die Tarifpartner verstärkt zu betrachten, mag nicht nur dabei helfen, zu erklären, wann Tarifverträge soziale Leistungen regulieren und finanzieren. Schließen Tarifpartner Verträge ab, die Umverteilung mittels Tarifpolitik finanzieren und organisieren, so kann das auch Rückwirkungen auf die Prozesspolitik und den Parteieneffekt in der Reform staatlich organisierter Umverteilungspolitik haben.

Bezüglich des Einflusses, den Modelle funktionaler Umverteilung und die industriellen Beziehungen auf den Parteieneffekt in Retrenchment-Prozessen ausüben, lassen sich in den vier Ländern zwei Muster erkennen: (1) In den Niederlanden und Däne- 
mark wird die tarifliche Altersvorsorge sowohl von den Sozialpartnern als auch von der Regierung in rentenpolitischen Reformprozessen strategisch genutzt. (2) In Frankreich und Deutschland erweisen sich die Systeme der tariflichen Altersvorsorge auf prozesspolitischer Ebene als relativ isoliert von Reformen der staatlichen Rentenpolitik. Durch die Kopplung zwischen funktionalem Modell der Umverteilung und territorialem Modell der Umverteilung auf Ebene der Prozesspolitik wird in den Niederlanden und Dänemark der Handlungsspielraum politischer Parteien bei Rentenreformen erhöht: Die Tarifrenten erlauben ihnen in rentenpolitischen Reformen mehr „zu können“ als in Deutschland und Frankreich. Dieser Artikel hat argumentiert, dass historische Prozesse der Institutionalisierung politischer und industrieller Staatsbürgerrechte als Weichensteller für Unterschiede in Parteieffekten fungieren können.

Die Rolle und Funktion von tariflicher Sozialpolitik in der gegenwärtigen Transformation von Wohlfahrtsstaaten sollte zum Gegenstand weiterer vertiefter empirischer Analysen werden. An die Analyse dieses Artikels schließen sich m.E. zwei Fragen an: (1) Aufgrund welcher treibenden Kräfte werden die industriellen Beziehungen im Zuge des Abbaus territorialer Umverteilung zu einem Träger sozialer Ordnung? (2) Wie kann eine historisch-institutionelle Analyse von Pfaden politischer und industrieller Opportunitätsstrukturen mit einer Analyse aktueller politischer Reformpräferenzen und -interessen sinnvoll verbunden werden, ohne einer Kontinuitätsillusion zu erliegen?

Ein Weg, sich diesen beiden Fragen zu nähern, könnte darin bestehen, wohlfahrtsstaatliche Retrenchmentprozesse als ein Phänomen inkrementellen institutionellen Wandels zu analysieren, und dabei Konzepte zu entwickeln, die es erlauben, in einem ersten Schritt zwischen Institutionenwandel als Reformpolitik und Institutionenwandel als Wandel „,by default“ zu differenzieren (Streeck 2005) und in einem zweiten Schritt die Rolle der Politik im Wandel von Institutionen konkreter zu spezifizieren. Der Wandel kann einfach mit Reformpolitik verbunden sein: Der Staat verleiht Verpflichtungsfähigkeit und setzt neben Recht auch Geld ein. Er verlagert staatliche Aufgaben in den intermediären Bereich der Verbände, weil die Präferenzen der Wähler es so wollen und/oder weil die strategischen Interaktionen zwischen politischen Akteuren dazu Möglichkeiten eröffnen. Der Wandel kann aber auch „by default“ geschehen. Das meint: Der Wandel kann auch ohne die Mobilisierung politischer Ressourcen und Handlungsfähigkeiten erfolgen, weil industrielle Beziehungen aufgrund historischer Institutionalisierungsprozesse eine Prädisposition für die Verlagerung von sozialer Sicherung in die Tarifpolitik aufweisen. Die Verbände machen die industriellen Beziehungen zur Quelle institutionellen Wandels. Sie nutzen die den industriellen Beziehungen inhärenten Verhaltensspielräume opportunistisch aus, indem sie die soziale Sicherung selbst regulieren. Wandel „by default“ bedeutet, dass das Eigeninteresse der Verbände und ihre kollektive Kreativität Wandel vorantreiben. Der Staat und die Politik können sich in der Tarifpolitik zurückhalten und die Dinge passieren lassen, die sowieso passieren, und zur gleichen Zeit in der staatlichen Sozialpolitik massiv Reformen und Kürzungen vorantreiben. Ist dies der Fall, so zeigt tarifliche Sozialpolitik eine interessante Facette der Neubestimmung des Verhältnisses zwischen Staat und Verbänden im Zuge staatlichen Sozialabbaus. Tarifliche Sozialpolitik ist eine überraschend kreative Antwort der Verbände auf die Erschöpfung des Staates in seiner Rolle als Mediator für den Interessenausgleich zwischen Arbeit und Kapital. Diese Antwort besteht darin, dass Verbände 
Staatsaufgaben, an denen ihnen besonders gelegen ist, in die industriellen Beziehungen und damit in eigene Regie übernehmen.

\section{Literatur}

Alber, Jens. 1987. Vom Armenhaus zum Wohlfahrtsstaat. Analysen zur Entwicklung der Sozialversicherung in Westeuropa. Frankfurt a.M.: Campus.

Allan, James P. and Lyle Scruggs. 2004. Political Partisanship and Welfare Reform in Advanced Industrial Societies. In: American Journal of Political Science 48(3). 496-512.

Amable, Bruno, Donatella Gatti and Jan Schumacher. 2006. Welfare-State Retrenchment. The Partisan Effect Revisited. In: Oxford Review of Economic Policy 22(3). 426-444.

Anderson, Karen M. 2006. The Netherlands: Political Competition in a Proportional System. In: Immergut, Ellen M., Karen M Anderson and Isabelle Schulze (eds.). West European Pension Politics. Oxford: OUP. 713-757.

Appendix. Table A10. Current Pension System in Denmark. In: Immergut, Ellen M., Karen M. Anderson and Isabelle Schulze (eds.). 2006. West European Pension Politics. Oxford: OUP.

Appendix, Table A15. Current Pension System in the Netherlands. In: Immergut, Ellen M., Karen. M. Anderson and Isabelle Schulze (eds.). 2006. West European Pension Politics. Oxford: OUP.

Armingeon, Klaus. 1994. Staat und Arbeitsbeziehungen. Ein internationaler Vergleich. Opladen: Westdeutscher Verlag.

ARRCO (Association des Régimes de Retraites Complémentaires). 2001. Supplementary Pension Schemes for Workers in France. Paris: ARRCO.

BAVC (Bundesarbeitgeberverband Chemie). 2004. Gemeinsame Presseerklärung von BAVC und IG BCE Chemie-Pensionsfonds mit 11\% Rendite. Wiesbaden, Hannover. 05.02.2004. www. bavc.de (18.05.2007).

Blatter, Joachim K., Frank Janning und Claudius Wagemann. 2007. Qualitative Politikanalyse. Eine Einführung in Forschungsansätze und Methoden. Wiesbaden: VS Verlag für Sozialwissenschaften.

Bradley, David, Evelyne Huber, Stephanie Moller, François Nielsen and John D. Stephens. 2003. Distribution and Redistribution in Postindustrial Democracies. In: World Politics 55(2). 193-228.

Brooks, Sarah M. 2002. Social Protection and Economic Integration: The Politics of Pension Reform in an Era of Capital Mobility. In: Comparative Political Studies 35(5). 491-523.

Caminada, Koen and Kees Goudswaard. 2005. Are Public and Private Social Expenditures Complementary? In: International Advances in Economic Research (11). 175-189.

ChemiePensionsfonds. 2007. Fast 40\% Wachstum beim ChemiePensionsfonds. Presseinformation vom 31.01.2007. München. https://www.chemiepensionsfonds.de/index.php?parm=frame\&p oint $=$ Unternehmen/Presse\&main=pg_presse (18.05.2007).

Conceição-Heldt, Eugénia da. 2006. France: The Importance of the Electoral Cycle. In Immergut, Ellen M., Karen M. Anderson and Isabelle Schulze (eds.). West European Pension Politics. Oxford: OUP. 150-199.

Cox, Robert H. 2001. The Social Construction of an Imperative. Why Welfare Reform Happened in Denmark and the Netherlands But Not in Germany. In: World Politics 53(3). 463-98.

DCA (Dienst Collectieve Arbeidsvoorwaarden). 1989. CAO-Afspraken 1988. Den Haag. 
Döring, Dieter. 2001. Kernsysteme der Alterssicherung und das Profil der betrieblichen Altersversorgung im europäischen Vergleich. In: Becker, Irene, Notburga Ott und Gabriele Rolf (Hrsg.). Soziale Sicherung in einer dynamischen Gesellschaft. Festschrift für Richard Hauser zum 65. Geburtstag. Frankfurt a.M.: Campus. 719-728.

Ebbinghaus, Bernhard. 1995. The Siamese Twins. Citizenship Rights, Cleavage Formation, and Party-Union Relations in Western Europe. In: Tilly, Charles (ed.). Citizenship, Identity and Social History. Cambridge: Cambridge University Press. 51-90.

Ebbinghaus, Bernhard. 2005. When Less is More. Selection Problems in Large-N and Small-N Cross-National Comparisons. In: International Sociology 20(2). 133-152.

Ebbinghaus, Bernhard. 2006. Reforming Early Retirement in Europe, Japan, and the USA. Oxford: OUP.

EIRO-France (European Industry Relations Observatory-France). 2004. EIRO, 2004: Questionnaire for EIRO Comparative Study on Occupational Pensions: France. http://eurofound. europa.eu/eiro/2004/04/word/fr0401105s.doc (18.05.2007).

EIRO-NL (European Industry Relations Observatory-Netherlands). 2004. EIRO, 2004: Questionnaire for EIRO Comparative Study on Occupational Pensions: The Netherlands. http:// eurofound.europa.eu/eiro/2004/04/word/n10401104s.doc (18.05.2007).

Emmenegger, Patrick. 2007. Parteien und Opportunitätsstrukturen: Die parteipolitischen Determinanten der Staats- und Sozialausgaben. 1980-2000. In: Zeitschrift für Sozialreform 53(1). 79-102.

Frericks, Patricia, Robert Maier and Willibrord de Graaf. 2006. Shifting the Pension Mix: Consequences for Dutch and Danish Women. In: Social Policy \& Administration 40(5). 475-492.

George, Alexander L. and Andrew Bennett. 2005. Case Studies and Theory Development. Cambridge (MA), London: MIT Press.

Gesamtmetall. 2007. Metallrente bilanziert hohes Wachstum - neue Angebote auch 2007. Pressemitteilung vom 29.01.2007. Berlin. http://www.metallrente.de/_content/pdf/presse/mitteilungen/070129_PM_Bilanz_MetallRente.pdf (18.05.2007).

Green-Pedersen, Christoffer. 2001. Welfare-state Retrenchment in Denmark and the Netherlands 1982-1998: The Role of Party Competition and Party Consensus. In: Comparative Political Studies 34(9). 963-985.

Green-Pedersen, Christoffer. 2004. The Dependent Variable Problem within the Study of Welfare State Retrenchment: Defining the Problem and Looking for Solutions. In: Journal of Comparative Policy Analysis 6(1). 3-14.

Green-Pedersen, Christoffer. 2006. Denmark A World Bank Pension System. In: Immergut, Ellen M., Karen M. Anderson and Isabelle Schulze (eds.). West European Pension Politics. Oxford: OUP. 43-51.

Green-Pedersen, Christoffer und Anders Lindbom. 2006. Politics within Paths. The Trajectories of Earningsrelated Pensions in Denmark and Sweden. In: Journal of European Social Policy 16(3). 245-258.

Haverland, Markus. 2001. Another Dutch Miracle? Explaining Dutch and German Pension Trajectories. In: Journal of European Social Policy 11 (4). 308-323.

Hemerijck, Anton and Philip Manow. 2001. The Experience of Negotiated Reform of the Dutch and German Welfare State. In: Ebbinghaus, Bernhard and Philip Manow (eds.). Comparing Welfare Capitalism. Social Policy and Political Economy in Europe, Japan and the USA. London: Routledge. 217-238.

Huber, Evelyne, Charles Ragin and John D. Stephens. 1993. Social Democracy, Christian Democracy, Constitutional Structure and the Welfare State. In: American Journal of Sociology 99(3). 711-749.

Jahn, Detlef. 2007. Was ist Vergleichende Politikwissenschaft? Standpunkte und Kontroversen. In: Zeitschrift für Vergleichende Politikwissenschaft 1(1). 9-27. 
Jørgensen, Carsten. 2004. New Collective Agreements Concluded in Industry. EIRO-Report. http://eurofound.europa.eu/eiro/2004/02/feature/dk0402104f.html (18.05.2007).

Kaar, Robbert van het and Marianne Grünell. 2004. Occupational Pensions and Industrial Relations. http://eurofound.europa.eu/eiro/2004/04/study/tn0404101s.html (18.05.2007).

Kenworthy, Lane and Jonas Pontusson. 2005. Rising Inequality and the Politics of Redistribution in Affluent Countries. In: Perspective on Politics 3(3). 449-471.

Kitschelt, Herbert. 2001. Partisan Competition and Welfare State Retrenchment: When Do Politicians Choose Unpopular Policies? In: Pierson, Paul (ed.). The New Politics of the Welfare State. Oxford: OUP. 265-302.

Kittel, Bernhard und Herbert Obinger. 2003. Parteien, Institutionen und Wohlfahrtsstaat. Politisch-institutionelle Determinanten der Sozialpolitik in OECD-Ländern. In: Obinger, Herbert, Uwe Wagschal und Bernhard Kittel. (Hrsg.). Politische Ökonomie: Demokratie und wirtschaftliche Leistungsfähigkeit. Opladen: Leske+Budrich. 355-384.

Korpi, Walter and Joakim Palme. 2003. New Politics and Class Politics in the Context of Austerity and Globalization: Welfare State Regress in 18 Countries, 1975-95. In: American Political Science Review 97(3). 425-46.

Lieberson, Stanley. 1991. Small N's and Big Conclusions: An Examination of the Reasoning in Comparative Studies Based on a Small Number of Cases. In: Social Forces 70(2). 307-320.

Mahoney, James. 2004. Comparative-Historical Methodology. In: Annual Review of Sociology 30. 81-101.

Marshall, Thomas H. 1964. Class, Citizenship, and Social Development. Garden City, New York: Anchor Books.

Mayntz, Renate. 2002. Zur Theoriefähigkeit makro-sozialer Analysen. In: Mayntz, Renate (Hrsg.). Akteure-Mechanismen-Modelle. Zur Theoriefähigkeit makro-sozialer Analysen. Frankfurt: Campus. 7-43.

Mill, John Stuart. 1874. A System of Logic. Rationative and Inductive. New York: Harper\&Brothers.

Myles, John and Paul Pierson. 2001. The Comparative Political Economy of Pension Reform. In: Pierson, Paul (ed.). The New Politics of the Welfare State. Oxford: OUP. 305-333.

Observatoire des Retraites. 2003. The French System. http://www.observatoire-retraites.org/versionanglaise/frenchsystem/Introduction.htm (18.05.2007).

Oorschot, Wim van. 1998. From Solidarity to Selectivity: the Reconstruction of the Dutch Social Security System 1980-2000. In: Social Policy Review (10). 183-202.

Øverbye, Einar. 1998. The Politics of Voluntary and Mandatory Pensions in the Nordic Countries. In: Labour 12(1). 169-198.

Palier, Bruno. 2006. The Long Good Bye to Bismarck? Changes in the French Welfare State. In: Culpepper, Pepper D., Peter A. Hall and Bruno Palier (eds.). Changing France. The Politics that Markets Make. Houndsmill: Palgrave Macmillan. 107-149.

Palier, Bruno. 2007. Tracking the Evolution of a Single Instrument Can Reveal Profound Changes: The Case of Funded Pensions in France. In: Governance. An International Journal of Policy, Administration and Institutions 20(1). 85-107.

Pierson, Paul. 1994. Dismantling the Welfare State? Reagan, Thatcher, and the Politics of Retrenchment. Cambridge: Cambridge University Press.

Polanyi, Karl. 1944. The Great Transformation. New York, Toronto: Rinehart.

Ragin, Charles. 1987. The Comparative Method. Berkeley: University of California Press.

Rein, Martin and John Turner. 2001. Public-Private Interactions: Mandatory Pensions in Australia, the Netherlands and Switzerland. In: Review of Population and Social Policy (10). 107-153.

Ross, Fiona. 2000. Beyond Left and Right: The New Partisan Politics of Welfare. In: Governance 13(2). 155-183. 
Schludi, Martin. 2005. The Reform of Bismarckian Pension Systems. A Comparison of Pension Politics in Austria, France, Germany, Italy and Sweden. Amsterdam: Amsterdam University Press.

Schulze, Isabelle and Sven Jochem. 2006. Germany: Beyond Policy Gridlock. In: Immergut, Ellen M., Karen M. Anderson and Isabelle Schulze (eds.). West European Pension Politics. Oxford: OUP. 660-710.

Scruggs, Lyle. 2006. The Generosity of Social Insurance. 1971-2002. In: Oxford Review of Economic Policy 22(3). 349-364.

Skocpol, Theda. 1979. States and Social Revolution. A Comparative Analysis of France, Russia, and China. Cambridge: Cambridge University Press.

Skocpol, Theda and Margaret Somers. 1980. The Uses of Comparative History in Macrosocial Inquiry. In: Comparative Studies in Society and History (22). 174-197.

Starke, Peter. 2006. The Politics of Welfare State Retrenchment: A Literature Review. In: Social Policy \& Administration 40(1). 104-120.

Streeck, Wolfgang. 2005. Institutional Change in France and Germany. A Workshop at the MPIfG. Cologne, 07.-08.10.2005. Mimeo.

Trampusch, Christine. 2005. Industrielle Beziehungen als Flexibilitätsressource korporatistischer Wohlfahrtsstaaten. Der Fall Sozialpolitik durch Tarifvertrag in den Niederlanden. In: Industrielle Beziehungen 12(2). 93-119.

Trampusch, Christine. 2007. Industrial Relations as a Source of Social Policy: A Typology of the Institutional Conditions for Industrial Agreements on Social Benefits. In: Social Policy \& Administration 41(3). 251-270.

Traxler, Franz. 1994. Collective Bargaining: Levels and Coverage. In: OECD Employment Outlook (July). 167-194.

Veil, Mechthild. 2004. Zukunft der Alterssicherung: Rentenpolitik und Rentenreform in Deutschland und Frankreich. In: Neumann, Wolfgang (Hrsg.). Welche Zukunft für den Sozialstaat? Reformpolitik in Frankreich und Deutschland. Wiesbaden: VS Verlag. 47-86.

Veil, Mechthild. 2005. Rentenreform 2003 in Frankreich: Mehr Flexibilität durch neue Steuerungsinstrumente. In: WSI-Mittelungen 1/2005. 25-31. 